\title{
Squamous Versus Adenosquamous Carcinoma in Gall Bladder
}

\author{
Neeti Nagar ${ }^{1}$, Meetu Agrawal ${ }^{2 *}$, Megha Tandon ${ }^{3}$ and Junaid Ansar ${ }^{4}$ \\ ${ }^{1}$ Department of Pathology, VMMC \& Safdarjung Hospital, New Delhi, INDIA \\ ${ }^{2}$ Department of Pathology, VMMC \& Safdarjung Hospital, New Delhi \\ ${ }^{3}$ Department of Surgery, VMMC \& Safdarjung Hospital, New Delhi, INDIA \\ ${ }^{4}$ Department of Surgery, VMMc \& Safdarjung Hospital New Delhi
}

\begin{abstract}
The commonest malignancy developing in gall bladder is an adenocarcinoma. Furthermore, the long-standing presence of gall stones is an inciting event in the development of malignancy. Squamous cell carcinomas have been known in the gall bladder. The squamous origin has been attributed to either of the two theories. It could be either squamous metaplasia in the lining of gall bladder and further malignant transformation or origin of a tumor as adenocarcinoma and its further differentiation towards squamous morphology. We support and demonstrate the latter morphology by means of this case.
\end{abstract}

\section{Keywords: Gall Bladder, Squamous Cell, Carcinoma}

\section{Introduction}

Gall bladder (GB) carcinomas are adenocarcinomas (AC) in the majority of cases. Adeno-squamous carcinoma (ASC) and pure squamous cell carcinoma (SCC) of the GB are rarely encountered. Incidence of pure squamous cell carcinoma in the gall bladder varies from less than $1 \%$ to $3 \%$ [1]; adenosquamous carcinoma accounts for less than $5 \%$ of all GB cancers [2]. Clinical presentation, behaviour and prognosis of these tumours is quite different from conventional adenocarcinomas. Their origin in the gall bladder which is lined by a prototypical secretory columnar epithelium could be explained either by metaplasia of the lining epithelium or by squamous differentiation of the neoplastic cells. Morphology in the present case was predominantly of a squamous cell carcinoma, but it highlighted an important theory of origin

\section{Case Report}

A 45 years old lady presented in surgical OPD with on and off pain in right upper abdomen and dyspepsia since last one month. There was history of loss of appetite and unintentional weight loss of $5 \mathrm{kgs}$ in last month. There was no history of jaundice, vomiting, altered bowel habits, blood in stools, bone pains, breathing difficulty. She was not aware of any cancers in the family or any co-morbidities or known drug allergies. General examination was within normal limits. No pallor, icterus, lymphadenopathy or oedema was present. Per abdominal examination reveal tenderness in right hypochondrium with palpable liver border. Liver span was $14 \mathrm{cms}$. USG abdomen reveal cholelithiasis with cholecystitis and GB sludge with asymmetric thickening of gall bladder wall CECT abdomen revealed chronic calculous cholecystitis with eccentric thickening involving fundus region of gall bladder in form of a necrotic mass, A $3.5 \times 2.6 \mathrm{~cm}$ calculus, closely abutting adjacent liver capsular surface and hepatic flexure of colon was noted.

After optimisation, the patient underwent radical cholecystectomy.

The gallbladder measured $7 \mathrm{~cm}$ in length with fundus measuring $4.2 \mathrm{cms}$ in maximum dimension. The external contour was smooth; cut surface showed a solid tumor occupying the fundus with no residual lumen. A large smooth stone measuring $4.1 \mathrm{~cm}$ was impacted at the neck. The tumour had a pearly white, firm appearance (Fig 1). Distance of tumor from the external surface was $0.2 \mathrm{~cm}$. Multiple representative sections from various areas revealed a moderately differentiated squamous cell carcinoma with focal presence of extracellular keratin (Fig 1b-c). Mild chronic inflammatory infiltrate was seen. No glandular component was seen in any of the initial sections. Further, extensive sampling was done from the junction between the normal mucosa and tumor almost exhausting the junction site. One of the sections revealed presence of a lining mucosa with villous configuration and moderate dysplasia. There was infiltration of neoplastic glands into the lamina propria. The adenocarcinoma, thus was a very small focus. Microscopic extent of the tumor invasion into the lamina propria was $2 \mathrm{~mm}$. Rest of the tumor showed squamous morphology (Fig 1d and inset). 
Wedge resection of the liver, lymph nodes at various levels and circumferential margin of the gall bladder was free of tumor. The area with squamous diffentiation showed positive staining for CK7 and p63 and no reactivity for CK20 and MOC31.

A diagnosis of squamous cell carcinoma with a small focus $(<1 \%)$ of adenocarcinoma was rendered. Pathological stage was pT2N0Mx.

\section{Discussion}

Gall bladder carcinomas are not so uncommon carcinomas with a fair incidence in North India [3]. Food habits, obesity and consistent presence of gall stones are factors to which the uphill trend of these malignancies may be attributed. Adenocarcinomas are the commonest histological type in majority cases; squamous differentiation of malignancy in the gall bladder is a rare occurrence. In most series the incidence is variable between $0.3-3 \%[1-3]$. Like conventional adenocarcinomas, SCCs have a female preponderance with F: $\mathrm{M}$ ratio of 3-4: 1. Most patients are elderly, in the sixth to seventh decade; our patient was a 45-year-old female.
When compared to and adenocarcinoma, the SCC is a highly malignant neoplasm presenting at an advanced stage in majority of the cases. Most of these tumors have higher propensity for loco-regional spread; so in many of these cases, debulking the tumor and palliative care is the only treatment option[4]. Etiopathogenesis for squamous differentiation in the gall bladder is not well defined. Because a normal gall bladder has no squamous epithelium, the source of origin is questionable. The most plausible theories involve either squamous metaplasia (metaplasia-dysplasia-carcinoma sequence) or squamous differentiation in an adenocarcinoma. [5]. Squamous cell carcinoma in our case could be explained by the latter theory as the junctional area in our tumor showed the origin of this tumor as an adenocarcinoma and thereafter the complete tumor underwent a squamous differentiation. Presence of gallstones and parasitic infestation are know to trigger squamous differentiation in initial pre-existing adenocarcinoma. This squamous differentiation subsequently grows rapidly and replaces the adenocarcinoma component to form pure squamous cell carcinoma. A large gallstone was impacted at the neck and could have elicited squamous differentiation in our case.

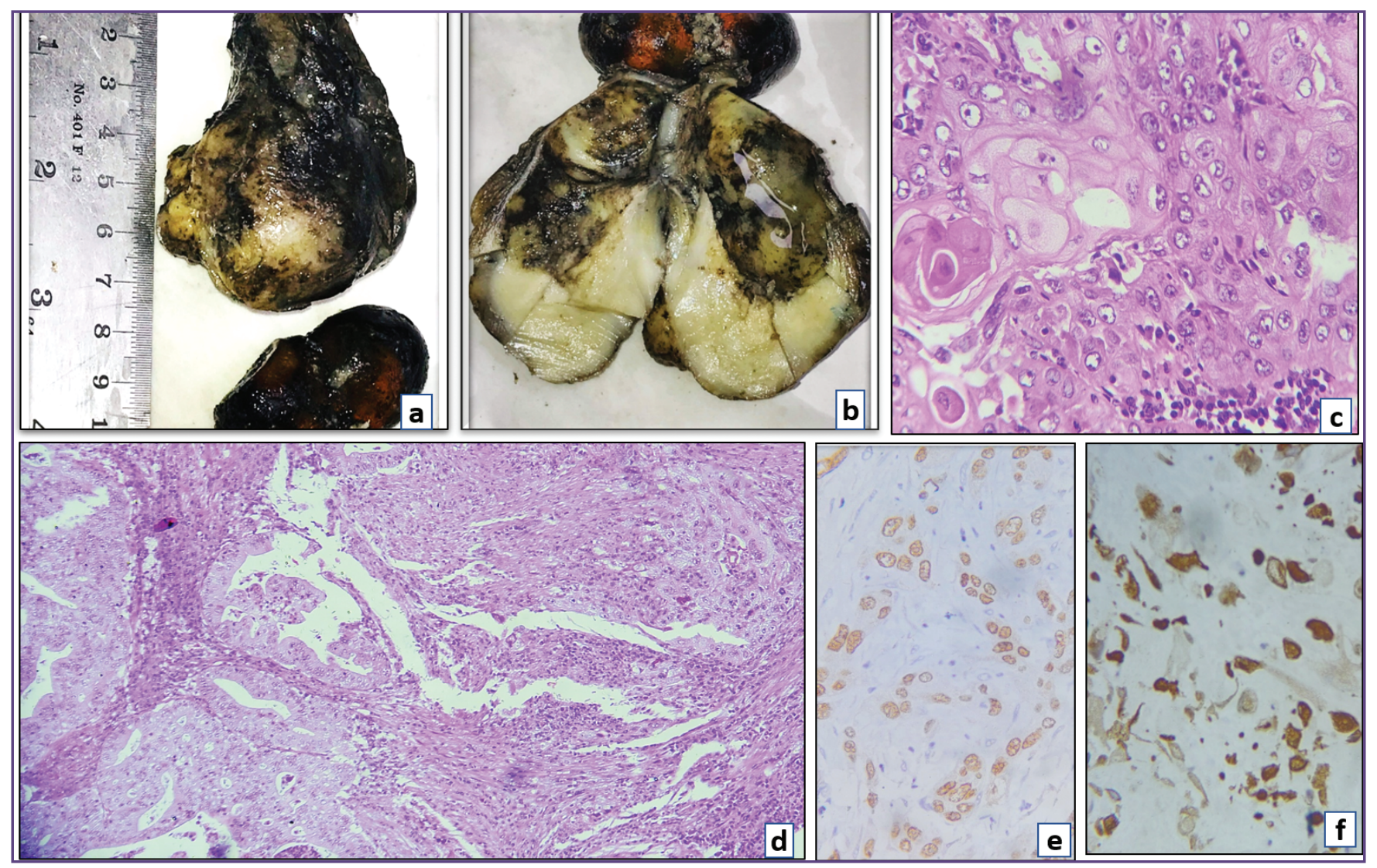

Fig. 1: (a) Specimen GB with (b) impacted cholesterol stone (c) Major portion with squamous differentiation, H \& E 40X (d) Junctional area with glandular and squamous components, H \& E 20X (e) p63 nuclear positivity (f) CK7 positivity in tumor islands. 
The concept of squamous cell carcinoma originating from preexisting metaplastic squamous epithelium appears unlikely; this kind of metaplasia virtually does not exist even in non-neoplastic conditions of gall bladder including chronic cholecystitis and cholelithiasis.

Furthermore, there is no definite cut-off percentage component of each element. Presence of $>25 \%$ squamous differentiation may be used to label a carcinoma as adenosquamous. [6] Converse criteria have not been defined. As $99 \%$ of the tumor area in this case showed a squamous morphology, we have preferred labelling it as a squamous cell carcinoma. Also the squamous morphology warrants a close follow up of the patient as these tumors have more propensity for local spread.Thus we have preferred to call it a higher grade tumor. Furthermore, tiny areas of adenocarcinoma were seen only after extensive sampling. As the small focus of glandular differentiation was infiltrating only upto the lamina propria, it did not have any further treatment implications other than cholecystectomy, which was already done in this case.

Gall stones appear to be a major risk factor in the carcinogenesis of carcinoma of any type but more so for squamous differentiation. [7] Approximately 90\% of squamous cell carcinoma cases invariably have cholelithiasis. Our case also had a gall stone. Presence of gall stone; especially a large impacted gallstone not only triggers squamous differentiation but is also responsible for pain being a major presenting symptom of SCCs in the gall bladder. In this regard, our patient was fortunate to have a large stone. This led to an early detection before the tumor could extend locally out of the gall bladder.

Other pathologies that have been associated with increased risk of gall bladder carcinomas include polypoidal lesions, adenomas, calcified porcelain gall bladder, cholecysto-enterior enteric fistulae, ulcerative colitis, adenomyomatosis, polyposis coli and anomalous connection between CBD and pancreatic duct. [8] None of these pathologies were found in our case.

Surgery is the primary treatment. However, due to the diagnosis of most of these tumors at an advanced stage, complete resection is not possible. This carcinoma is rarely detected at an early resectable stage. In this case however, early presentation due to severe pain, free circumferential margin and absence of loco-regional spread facilitated a good surgical outcome.

\section{Conclusion}

Knowledge of various types of gall bladder cancers and their precursor lesions can aid in understanding the behavior and treatment of both usual and unusual types of gall bladder cancers. SCCs of the gall bladder often present with large, bulky tumors with involvement of adjacent organs. Careful sonographic examination may help to decrease the mortality associated with this disease enabling early detection at an earlier stage. In spite of progress in surgical techniques and adjuvant chemotherapy, the prognosis of GBSCC remains poor.

\section{Acknowledgements}

We acknowledge the work of our histopathology technical staff Mr Rana, who patiently helped us in this case

\section{Reference}

1. Perisetti A, Raghavapuram S, Tharian B, et al. Pure Squamous Cell Carcinoma of the Gallbladder Masquerading as a Hepatic Mass. Cureus. 2018;10(1):e2011. Published 2018 Jan 1. doi:10.7759/cureus.2011

2. Bagdasaryan R, Miroshnichenko H. Adenosquamous cell carcinoma of the gallbladder. Images in medicine 2011;94(1):19.

3. Lal M, Raheja S, Bhowmik K (2018) Carcinoma Gallbladder-Epidemiological Trends in a Tertiary Hospital in North India. Arch Surg Oncol 4: 131.

4. Hosseinzadeh M, Shokripur M, Salahi H. Primary Pure Squamous Cell Carcinoma of Gallbladder Presenting as Acute Cholecystitis. Iran J Med Sci. 2012;37(4): 271-273.

5. Roppongi T, Takeyoshi I, Ohwada S, Sato Y, Fujii T, Honma $\mathrm{M}$, et al. Minute squamous cell carcinoma of gall bladder: A case report. Jpn J Clin Oncol 2003;30:43-5.

6. Singh S, Ansari MA, Narayan G. Pathobiology of gall bladder cancer. J Scientific Research 2012;56:35-45.

7. Schwrtz SL, Shires E, Spencer FC, Daly JM, Fisher JE, Galloway AC. Principles of surgery. 7 th ed, New York: McGraw-Hill; 2006.

8. Bai PJ, Srujana S, Quadri SS, Kumari NS. Squamous cell carcinoma of gall bladder: A rare case. Med J DY Patil Univ $2013 ; 6: 455-8$

*Corresponding author:

Dr Meetu Agrawal, Department of Pathology, VMMC \& Safdarjung Hospital, DELHI, INDIA

Phone: +917838147603

Email: meetu.drmeetuagrawal@gmail.com

Financial or other Competing Interests: None. 